\title{
Clinical significance and diagnostic value of serum NSE, CEA, CA19-9, CA125 and CA242 levels in colorectal cancer
}

\author{
HAI LUO, KEXIN SHEN, BO LI, RUIQI LI, ZEMING WANG and ZHONGSHI XIE \\ Department of Gastrointestinal Colorectal and Anal Surgery, China-Japan Union Hospital of Jilin University, \\ Changchun, Jilin 130033, P.R. China
}

Received August 7, 2019; Accepted January 17, 2020

DOI: $10.3892 / \mathrm{ol} .2020 .11633$

\begin{abstract}
The present study investigated the value of combinations of five specific tumor biomarkers for the diagnosis of colorectal cancer (CRC): Neuron-specific enolase (NSE), carcinoembryonic antigen (CEA), cancer antigen (CA)19-9, CA125 and CA242. Associations between these markers and clinicopathological characteristics (including the Tumor-Node-Metastasis stage) were also assessed. Serum levels of the 5 markers were compared between 358 patients with CRC and 298 healthy individuals (CRC and control group, respectively). The NSE concentration of the CRC group was significantly higher compared with the control. Furthermore, patients at clinical stage III+IV exhibited significantly higher NSE levels compared with those at stage I+II. The serum NSE level of $\mathrm{N}^{+}$patients was significantly higher compared with the $\mathrm{N}^{-}$group, and the NSE level of $M_{1}$ patients was significantly compared with the $M_{0}$ group. NSE level was also significantly associated with tumor stage, lymph node metastasis, distant metastasis and hematochezia. The area under the receiver operating characteristic curve (AUC) for NSE in CRC was 0.766, which was significantly higher than that of the other four markers, which ranged from 0.560-0.682. The AUC of NSE, CEA, CA19-9, CA125, CA242 combined was significantly higher compared with any of the markers individually (range, 0.796-0.858). Therefore, serum NSE may be a good clinical tool for the auxiliary diagnosis of colorectal cancer. Besides, the combination of NSE, CEA, CA19-9, CA125 and CA242 was significantly more sensitive compared with NSE alone. Thus, the combined detection of the 5 tumor markers may be more useful for the diagnosis of CRC.
\end{abstract}

Correspondence to: Professor Zhongshi Xie, Department of Gastrointestinal Colorectal and Anal Surgery, China-Japan Union Hospital of Jilin University, 126 Xian Tai Street, Changchun, Jilin 130033, P.R. China

E-mail: surgeonxzs@163.com

Key words: colorectal cancer, neuron-specific enolase, tumor markers, diagnosis, prognosis

\section{Introduction}

Cancer represents a major public health issue worldwide, and is a leading cause of mortality (1). Globally, colorectal cancer (CRC) is the third most frequent cancer type, with $>1.4$ million new cases and $>690,000$ mortalities annually (2). For patients with CRC, survival time is significantly dependent on the stage of cancer upon diagnosis, with 5 year survival rates of $\sim 90$, 70 and $13 \%$ for localized, regional and distantly metastatic stages, respectively (3). CRC is slow to progress and noticeable symptoms, such as bloody stools (hematochezia), abdominal pain, fatigue and appetite loss (4). Therefore, improving the prognosis for patients with CRC largely depends upon early and accurate diagnosis.

Currently, the most accurate method of diagnosis of $\mathrm{CRC}$ is colonoscopy combined with pathological biopsy (5); however, these tests are invasive and expensive, potentially life-threatening complications, and patient compliance is poor (6). Inexpensive and non-invasive methods have been proposed, such as fecal occult blood-based screening, but both the sensitivity and specificity are lower than colonoscopy $(7,8)$. Recently, certain researchers have proposed that an exosome vesicle containing numerous specific antigen proteins, DNA, long non-coding RNA (lncRNA) and microRNA (miRNA) may have diagnostic value in CRC (9-11). However, such a test would be clinically impractical as the implementation may be complex, time-consuming, expensive, and the sensitivity and specificity may also be low.

Serum tumor biomarkers may serve not only for auxiliary diagnosis of CRC, but also as tools for estimating survival and prognosis. Notably, commonly used tumor markers for the diagnosis and assessment of patients with CRC are carcinoembryonic antigen (CEA), cancer antigen (CA)19-9, CA125 and CA242 $(12,13)$. CEA may be the best biomarker for differential diagnosis of malignant tumors, disease monitoring and evaluation of efficacy, relative to the other markers as serum CEA levels are an important prognostic factor and indicator of therapeutic effect and recurrence in patients with CRC $(14,15)$. However, CA19-9, CA125 and CA242 have also been used as indicators for CRC diagnosis, postoperative surveillance and the monitoring of treatment effects (16-18).

Due to the highly heterogeneous nature of CRC, a single tumor marker is unlikely to represent an accurate diagnostic standard with sufficient sensitivity or specificity for all cases. 
Recent studies have indicated that combining multiple tumor markers may improve the accuracy of diagnostic and prognostic evaluations. For example, CEA combined with CA242 achieved significantly higher sensitivity compared with either alone (13). Furthermore, Wang et al (18) demonstrated that CEA, CA19-9 and CA242, when analyzed together, improved the accuracy of prognostic prediction in patients with CRC who underwent surgery.

Serum neuron-specific enolase (NSE) is a cell-specific isoenzyme of the glycolytic enzyme enolase, first discovered in brain tissue extracts (19). NSE has been widely used as a clinical biomarker for diagnosis and prognosis of various benign or malignant diseases. For example, human serum NSE concentration is directly proportional to the extent of brain damage caused by conditions such as cerebral ischemia, and is therefore an important biological marker for severe brain injury (20,21). Additionally, NSE is a highly specific marker for neuronal and peripheral neuroendocrine cells, it was the first marker used to identify neuroendocrine cells, and as such is particularly used for the diagnosis of malignant tumors (22). Serum NSE level is associated with melanoma, seminoma, renal cell carcinoma, Merkel cell tumor, carcinoid tumor, dysgerminoma, immature teratoma and malignant pheochromocytoma, particularly in small cell lung cancer (SCLC), but also in other cancer types (23). However, the potential value of NSE in CRC is yet to be elucidated.

It has been hypothesized that that there is a high positive rate for NSE in patients with CRC, and that NSE level may be associated with tumor staging. Therefore, the present study investigated the value of NSE for the diagnosis of CRC, and determined its association with tumor staging and potential value for prognostic evaluation. The present study also evaluated the value of combining the detection of NSE, CEA, CA19-9, CA125 and CA242 for the diagnosis of CRC.

\section{Materials and methods}

The Ethics Standards Committee of China-Japan Union Hospital of Jilin University (Jilin, China) approved the protocol of the present study. All patients provided written informed consent and the experiments were performed in accordance with the relevant guidelines and regulations of the aforementioned hospital.

Patients and samples. Tumors were staged based on the Tumor-Node-Metastasis (TNM) classification of the American Joint Committee on Cancer Staging (eighth edition) (24).

The patient CRC group comprised 358 patients who were hospitalized between July 2017 and March 2019 at The China-Japan Union Hospital of Jilin University (Jilin, China), with CRC of the rectum ( $n=193)$, left colon $(n=87)$ or right colon $(\mathrm{n}=78)$. The CRC group comprised 218 men and 140 women, aged between 27 and 85 years (median \pm SD, 61.1 \pm 11.9 years). None of the patients had received preoperative neoadjuvant chemoradiotherapy or any other treatment for their tumor. For analyses, the CRC group was stratified according to tumor site (rectum, left or right colon) or clinical stage (I+II or III+IV).

All the included patients underwent surgery. Serum samples were collected preoperatively. Patients with stage I-III cancer were treated with radical surgery, while patients at stage IV received either radical or palliative surgery. Postoperative pathological examinations were performed to confirm a diagnosis of CRC in all patients. All included patients had complete clinical and pathological data. In addition, the control group consisted of 298 healthy volunteers (age range, 20-75 years; median $\pm \mathrm{SD}, 55.8 \pm 8.9$ years, 158 men and 140 women), who were free of any vital infections or gastrointestinal disease.

Detection of serum tumor markers. Fasting venous blood samples $(2 \mathrm{ml})$ were collected from the elbow of all patients between 06:00 and 07:00 a.m. on the second day following admission and submitted to the Central Research Office of The China-Japan Union Hospital of Jilin University for quantitative analysis of the relevant biomarkers. Blood samples were centrifuged at room temperature, at 2,000 x g for $5 \mathrm{~min}$, and the supernatant was added to the corresponding tumor kit for detection (Luminex Ltd.).

All lab tests were performed in accordance with the standard operating procedures. The experiments were performed on the day of sample collection, and the reports were used to guide the clinical decisions of the physicians. The cut-off values for the serum markers were in accordance with the recommendations of the manufacturer (Tellgen Corporation); specifically, 25.00 and $5.00 \mathrm{ng} / \mathrm{ml}$ for NSE and CEA, respectively, and 37.00, 35.00 and $20.00 \mathrm{U} / \mathrm{ml}$ for CA19-9, CA125 and CA242, respectively. An overview of the 5 markers and their association with CRC is displayed in Table I.

Statistical analysis. Associations between the preoperative serum levels of the 5 tumor biomarkers and clinicopathological characteristics were analyzed using Pearson's chi-squared $\left(\chi^{2}\right)$ test or Fisher's exact probability test. Comparisons between the serum levels of the 5 tumor biomarkers in the CRC and control groups were evaluated using the Mann-Whitney U test. The areas under the receiver operating characteristic (ROC) curve (AUC), 95\% confidence interval (CI), and Youden's index (sensitivity + specificity -1) were calculated for each tumor biomarker, and the combination of all 5 markers. Logistic regression was used to analyze the diagnostic power of each of the 5 markers, and the combination of the markers, for the CRC group and its various subgroups; the Hosmer-Lemeshow goodness-of-fit test was used to assess the model. The AUCs of the combination test and the individual biomarkers were compared via the $\mathrm{Z}$ test using the MedCalc V15.2 software. $\mathrm{P}<0.05$ was considered to indicate a statistically significant difference. All of the aforementioned statistical analyses were performed using SPSS v18.0 (SPSS, Inc.).

\section{Results}

Serum levels of the 5 tumor markers in the control and CRC groups. The preoperative serum levels of the 5 markers in the patients with CRC were significantly higher compared with the control group. Specifically, the median levels of serum NSE and CEA in the CRC (and median of control) group were 21.15 (15.63) and 3.01 (1.77) ng/ml, respectively; the median levels of serum CA19-9, CA125 and CA242 were 11.08 (9.54), 12.49 (11.07), and 5.79 (3.74) U/ml (Table II).

The CRC group was further stratified according to tumor site and for each subgroup (rectum, left colon and right colon), 
Table I. An overview of the association between biomarkers investigated in the present study and CRC.

\begin{tabular}{ll}
\hline Marker & \multicolumn{1}{c}{ Rationale } \\
\hline NSE & $\begin{array}{l}\text { NSE is a tumor marker widely used in the diagnosis of SCLC, but the association between } \\
\text { serum NSE levels and CRC are out of date and limited } \\
\text { CEA is an important prognostic factor and an indicator of the therapeutic effect and } \\
\text { recurrence in patients with CRC }\end{array}$ \\
CA199 & $\begin{array}{l}\text { Indications are that elevated levels may be informative for some CRC cancers } \\
\text { CA125 is more sensitive for mucin-type ovarian cancer, and its combination with CA125 can } \\
\text { SA125 }\end{array}$ \\
CA242 & $\begin{array}{l}\text { CA242 combined with other mantivers may improve early diagnosis of CRC, and the accuracy } \\
\text { of prognostic prediction in surgically treated patients with CRC }\end{array}$ \\
\hline
\end{tabular}

NSE, neuron-specific enolase; CEA, carcinoembryonic antigen; CA199, cancer antigen 19-9; CA125, cancer antigen 125; CA242, cancer antigen 242; CRC, colorectal cancer; CI, confidence interval.

the serum levels of NSE, CEA, CA12 and CA242 were significantly higher compared with the control (Table II). Notably, the serum concentration of CA19-9 was higher in the rectum and left colon cancer subgroups compared with the control, but the levels of the right colon cancer subgroup and control were not significantly different.

Associations between biomarkers and clinicopathological parameters. The 5 markers were investigated for associations with pathological parameters of CRC (Table III). Results of the $\chi^{2}$ tests indicated that the serum NSE level was significantly associated with hematochezia and the N, M and pathological (p) TNM stage, but not on any of the following: Vascular invasion, nerve infiltration, tumor differentiation, tumor location, tumor size, sex or age.

The serum CEA level was significantly associated with vascular invasion, nerve infiltration, tumor differentiation, and tumor size and stage; however, there was no association with age, sex or hematochezia. Serum CA19-9 was significantly associated with nerve infiltration, $\mathrm{N}$ stage and pTNM staging $(\mathrm{P}<0.05)$. However, there was no significant association between positive serum CA19-9 levels and M stage, vascular infiltration, tumor differentiation, tumor location and sex $(\mathrm{P}>0.05)$. Similarly, there were no significant statistical differences for preoperative, or sex. The serum CA125 level was associated with the $\mathrm{N}$ stage and pTNM staging, but not vascular invasion, nerve infiltration, tumor differentiation or hematochezia. Furthermore, serum CA242 was significantly associated with lymph node metastasis, nerve invasion and T, N and pTNM staging, but not with $\mathrm{M}$ stage, tumor differentiation, vascular invasion, hematochezia or sex.

Associations between biomarkers and clinical stage of disease. The CRC group was stratified by the clinical stages (I+II or III+IV), and the positive rates of the 5 tumor markers were calculated (Fig. 1) and were revealed to increase with the clinical stage, with significant differences in serum levels of each marker between the two subgroups. In addition, the rate of positivity for the five tumor markers were significantly higher in patients with lymph node metastasis compared with those without. However, only the rate of positivity for NSE and
CEA were significantly higher in patients with distant metastasis compared with those without distant metastasis.

Logistic regression and $R O C$ curve analyses. For the CRC group, ROC curves were constructed for each of the 5 tumor markers, and their combination (Fig. 2). Overall, for the 358 patients the areas under the ROC curves (AUCs) for each of the markers were as follows: NSE, 0.766; CEA, 0.682; CA19-9, 0.560; CA125, 0.590; and CA242, 0.651. Notably, the AUC for the combination of all 5 markers was 0.827 .

ROC curves were also analyzed for each of the CRC subgroups according to tumor site (rectum, left colon and right colon). For the rectal subgroup, the AUCs of the NSE, CEA, CA19-9, CA125, CA242 were: 0.794, 0.686, 0.562, 0.576 and 0.662 , respectively, and the AUC for the combination of all 5 markers was 0.858 . In patients with left colon cancer, the AUCs of the respective 5 markers and their combinations were: $0.777,0.653,0.584,0.582$ and 0.653 . In patients with right colon cancer the respective AUCs were: 0.708, 0.702, $0.529,0.637$ and 0.622 .

The Hosmer-Lemeshow test was conducted to verify the appropriateness of the logistic regression model for analyzing $\mathrm{CRC}$, and the various subtypes of tumor site in CRC. The results were as follows: $\mathrm{CRC}, \chi^{2}=6.326, \mathrm{P}=0.611$; rectal $\mathrm{CRC}$, $\chi^{2}=4.874, \mathrm{P}=0.771$; left colon $\mathrm{CRC}, \chi^{2}=9.771, \mathrm{P}=0.281$; and right colon $\mathrm{CRC}, \chi^{2}=9.082, \mathrm{P}=0.335$.

The AUCs for predicted probability of CRC associated with each tumor marker and logistic regression curves were constructed. Among the 5 tumor markers analyzed individually, the AUC of NSE was the highest (Table IV). Yet, the AUC of the combination of all markers exceeded that of any individual marker. For the diagnosis of CRC, both NSE and CA242 had high sensitivity (63.41 and 66.2, respectively), but the specificity of NSE was higher (79.53 vs. 59.73). For each of the tumor site subgroups, the sensitivity of the combined tumor markers was highest, but the specificity was lower.

The AUCs of the individual biomarkers and their combination were compared using a $\mathrm{Z}$ test for the CRC group, and the tumor location subgroups. For the CRC group overall, in comparison with the combination of all markers the $Z$ values were as follows (all $\mathrm{P}<0.01$ ): NSE, 5.47; CEA, 7.359; CA19-9, 11.159; CA125, 10.476; and CA242, 7.865. In patients with 

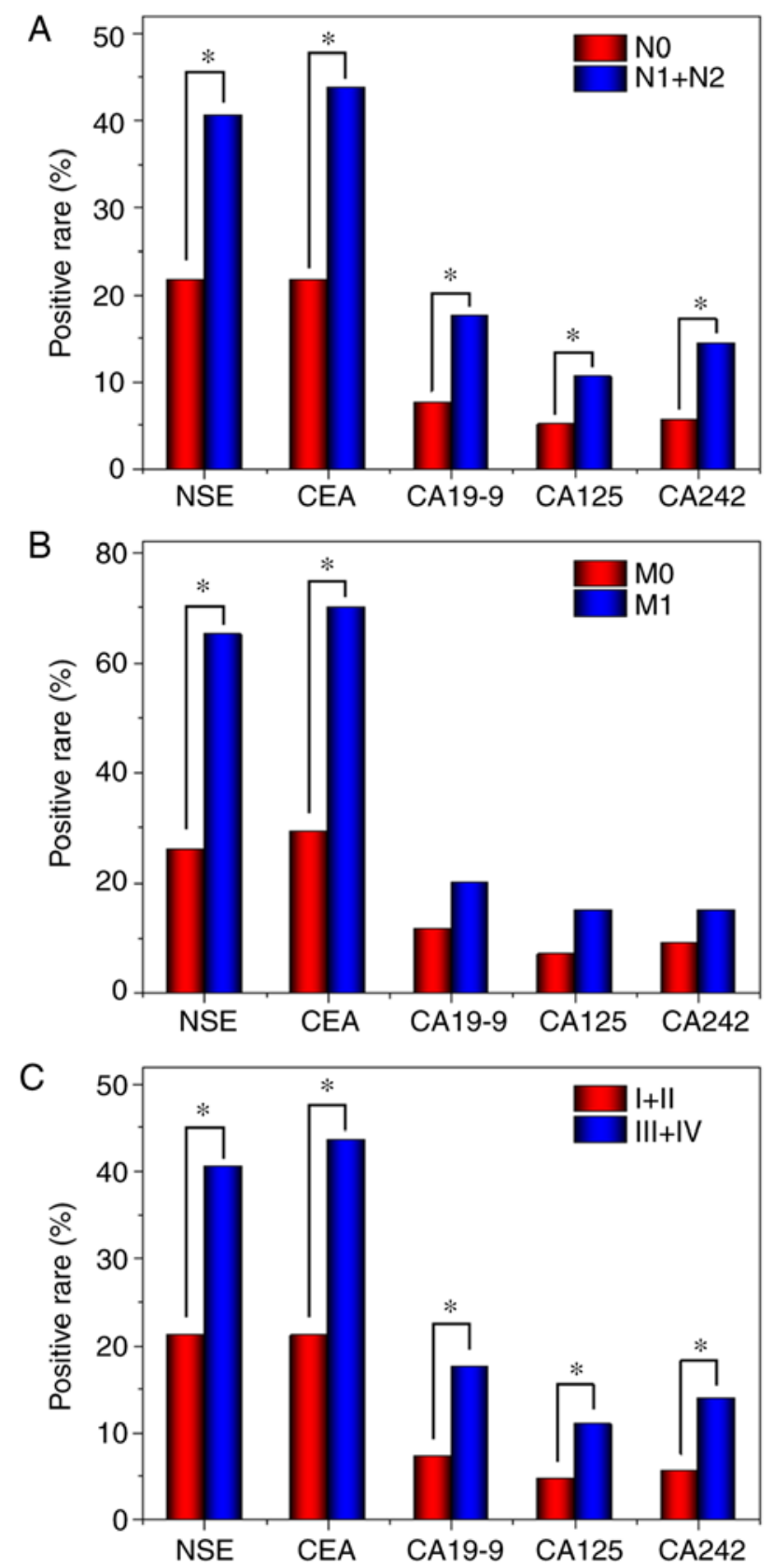

Figure 1. Percentages of patients testing positive for the 5 tumor markers, according to (A) local lymphatic (N stage); (B) distant metastasis (M stage); and (C) pTNM stage. ${ }^{*} \mathrm{P}<0.05$ vs. control group. NSE, neuron-specific enolase; CEA, carcinoembryonic antigen; CA199, cancer antigen 19-9; CA125, cancer antigen 125; CA242, cancer antigen 242; CRC, colorectal cancer; pTNM, pathological tumor node metastasis.

rectal cancer, the $\mathrm{Z}$ values for NSE, CEA, CA19-9, CA125 and CA242 compared with the combination test were 4.997, 7.007, $10.123,9.785$ and 7.451 , respectively (all $\mathrm{P}<0.01$ ). In patients with left colon cancer, the respective $Z$ values were 2.772 , 4.864, 5.976, 6.191 and 4.887 (all $\mathrm{P}<0.01$ ), compared with the combination test.

\section{Discussion}

Serum tumor markers are considered as biological indicators detected from the serum or plasma of suspected tumor patients. The increase of such indicators indicates tumor 

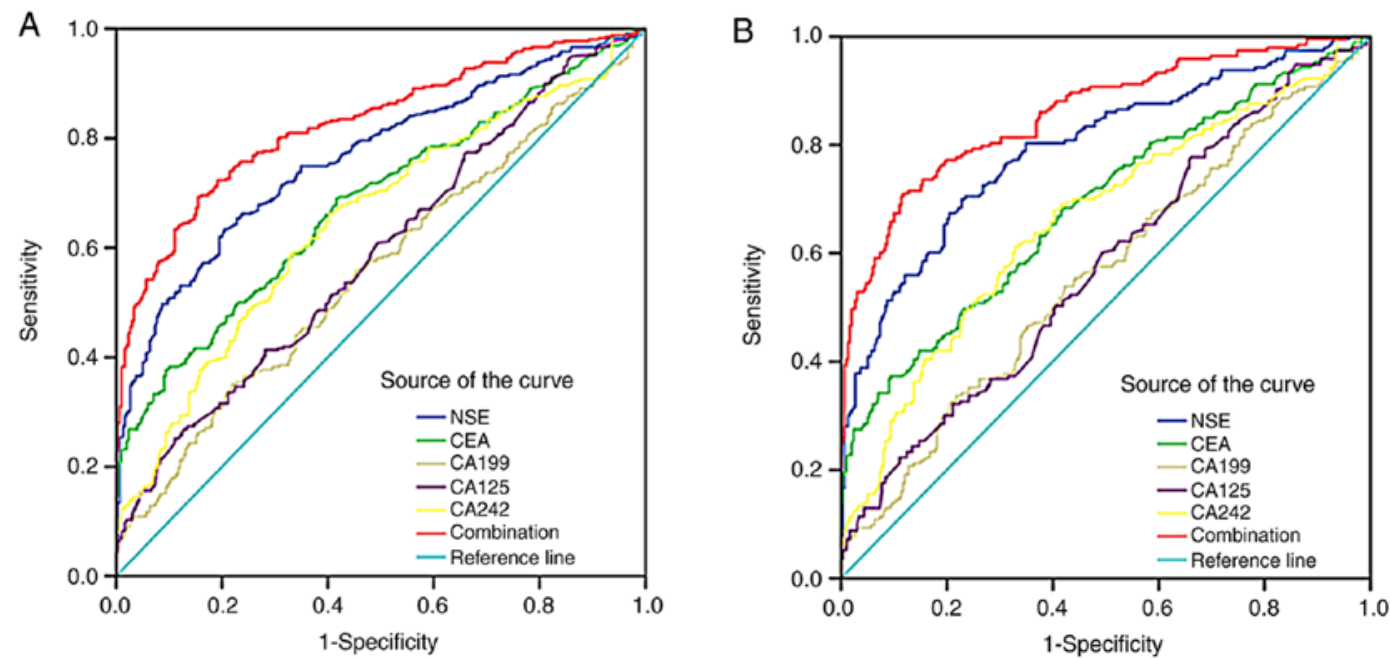

Colorectal cancer
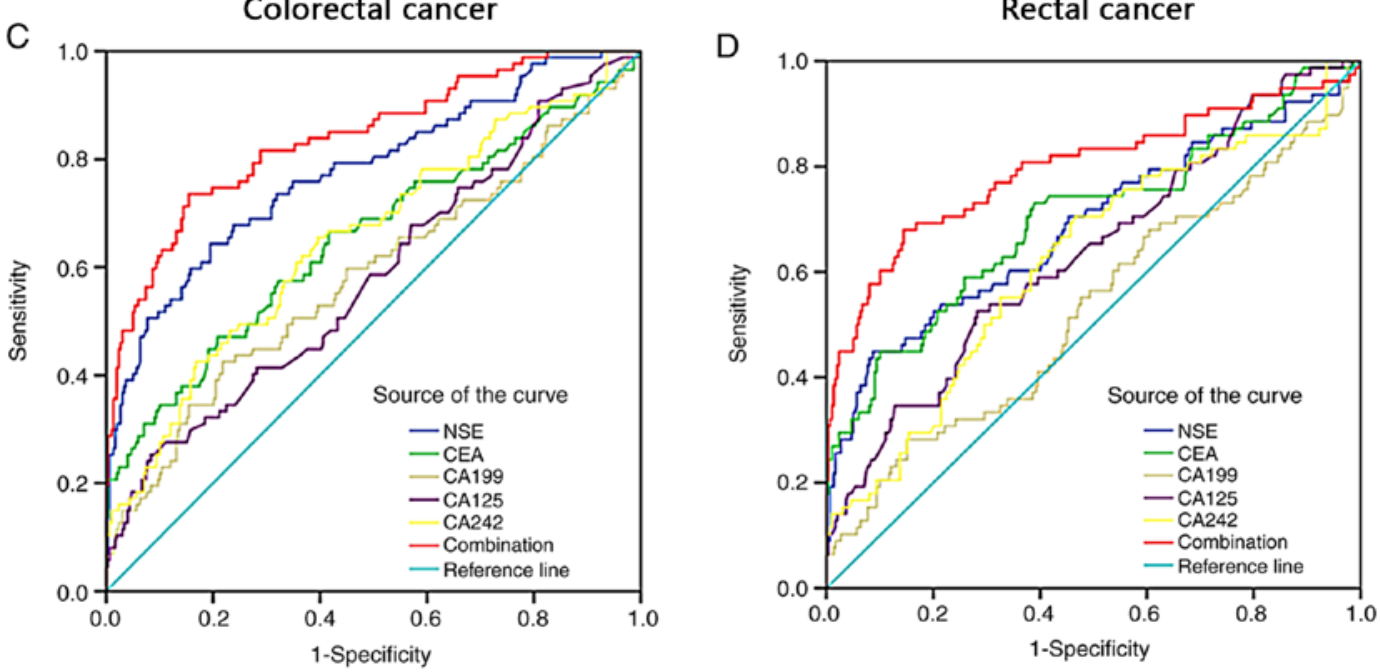

Left colon cancer

Right colon cancer

Figure 2. Diagnostic value of combined detection of the tumor markers NSE, CEA, CA19-9, CA125 and CA242 for CRC is superior to each independent tumor marker. ROC curves of single NSE, CEA, CA19-9, CA125, CA242 and the combination in predicting (A) colorectal cancer; (B) rectal cancer; (C) left colon cancer; and (D) right colon cancer. NSE, neuron-specific enolase; CEA, carcinoembryonic antigen; CA199, cancer antigen 19-9; CA125, cancer antigen 125; CA242, cancer antigen 242; CRC, colorectal cancer.

existence, facilitating pathological analysis and evaluation of tumor development (25). Serum tumor biomarkers are useful for choosing treatment strategies, particularly when the markers are convenient and economically efficient to detect (26). CEA is often secreted by tumors located in the digestive tract and is the most widely used CRC marker (27). CEA has good specificity and sensitivity for screening CRC and is a valuable tool for evaluating the prognosis of patients with CRC (28). The understanding of malignant tumors and their associated serum markers has increased in previous years, and this has indicated the potential of biomarkers in facilitating improvements to the diagnosis and evaluation of treatment effect and prognosis (29). However, the sensitivity and specificity of a single serum tumor marker for CRC is not precise, making it necessary to select and combine a variety of markers to improve accuracy. Notably, Gao et al (30) identified a combination of serum markers for the diagnosis of CRC, and also to appraise tumor status to guide treatment, evaluate curative effect and predict prognosis.
NSE is a tumor marker widely used in the diagnosis of SCLC. In patients with SCLC, serum NSE levels are significantly elevated (31), but they are also higher in patients with non-small cell lung cancer (NSCLC) and other types of tumor (32). The association between serum NSE levels and CRC also has been previously reported $(33,34)$. In the present study, $30.16 \%$ of patients with CRC tested positive for NSE, which was lower than the rate for CEA (31.56\%), but significantly higher compared with CA19-9 (12.0\%), CA125 (7.5\%) and CA242 (9.5\%). The positive rate for NSE in patients with CRC is similar to that of CEA and consequently, it was hypothesized that NSE may represent a biomarker associated with colorectal tumor growth that may be used for the diagnosis and evaluation of CRC progression.

In the present study, the association between serum NSE values and clinical stage was investigated with the intention of determining whether serum NSE may be used for the auxiliary diagnosis and evaluation of tumor progression in patients with CRC. Initially, the serum NSE levels of 


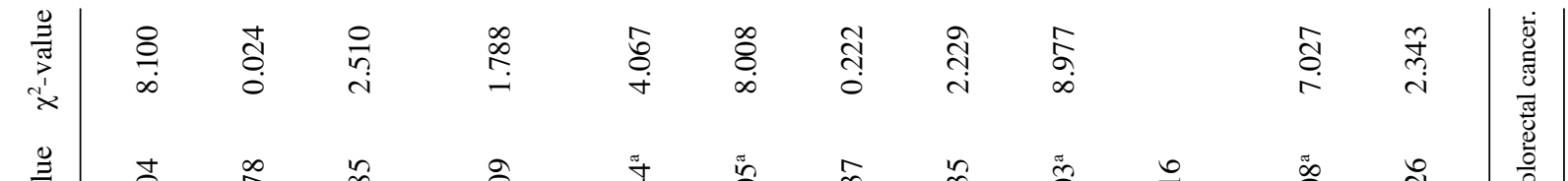

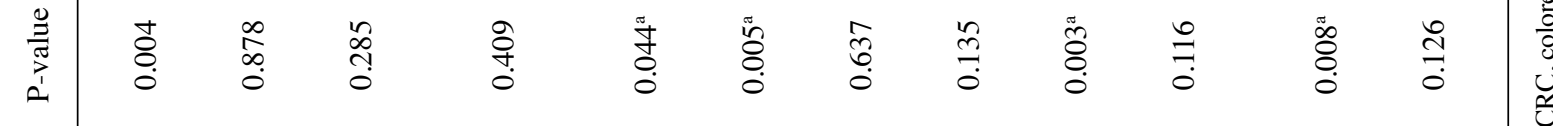

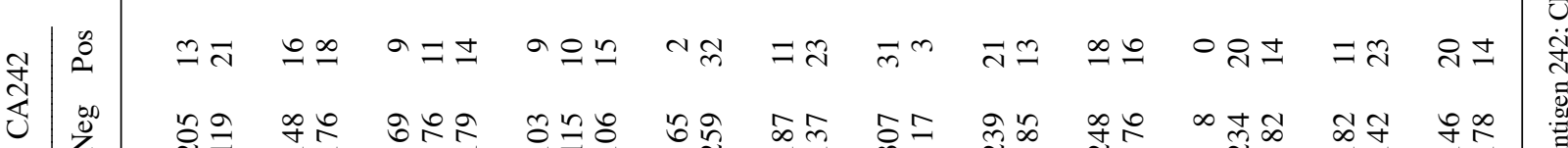

Uิ

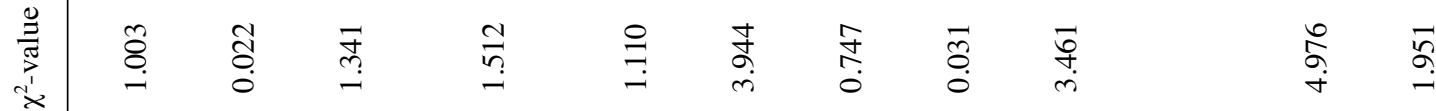

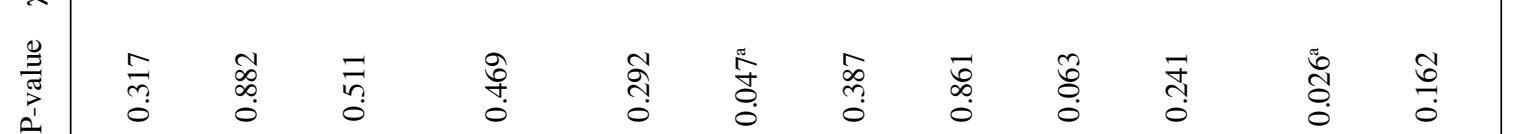

I)

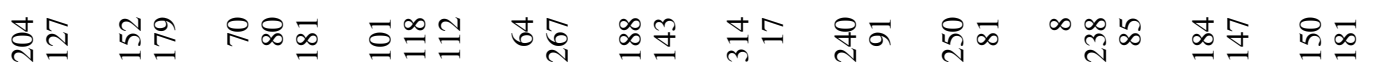

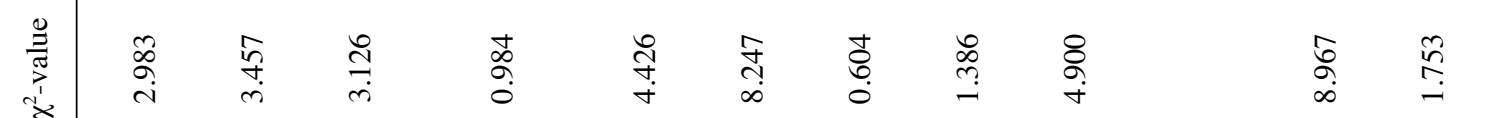

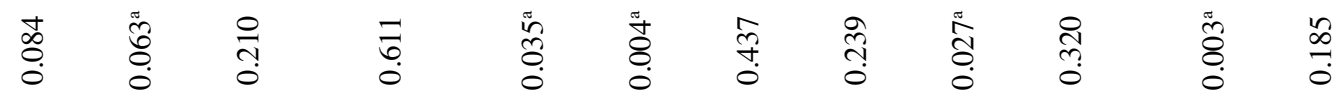

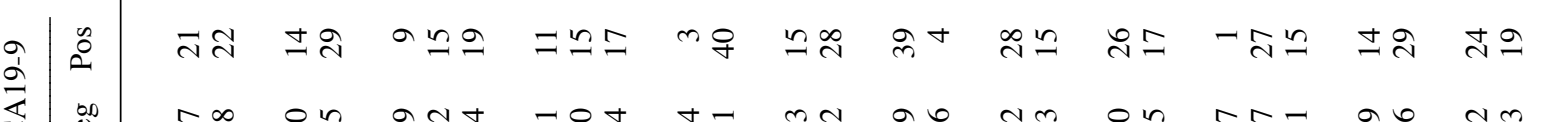

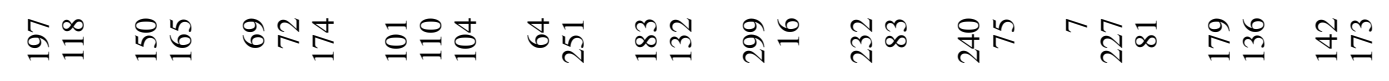

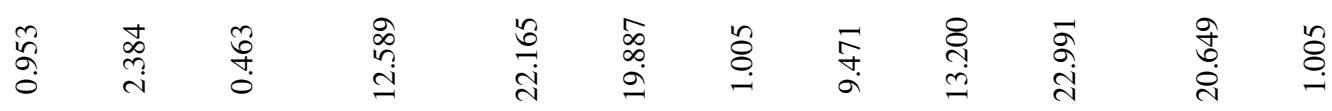

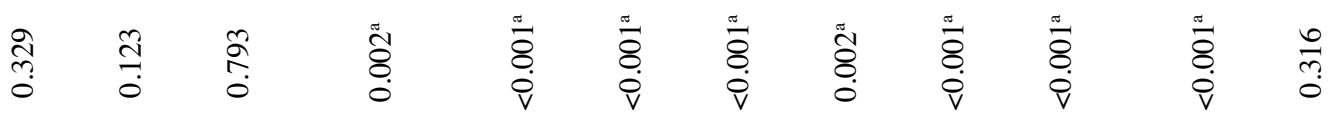

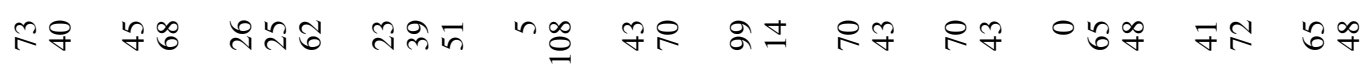

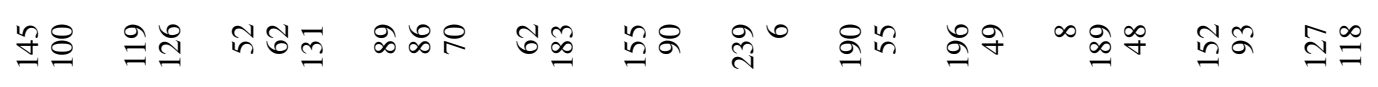

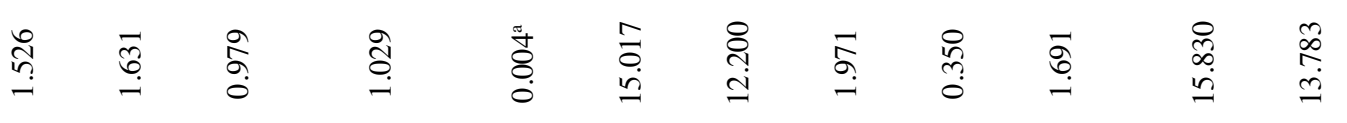

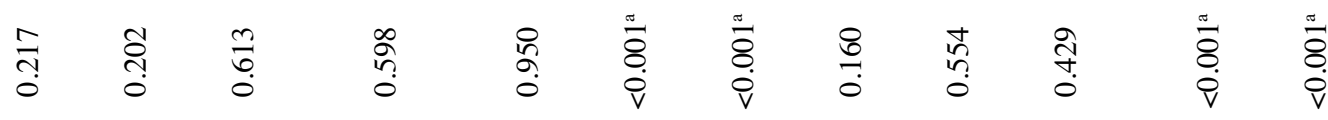

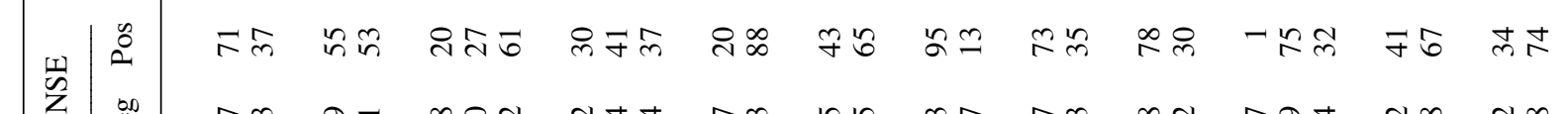

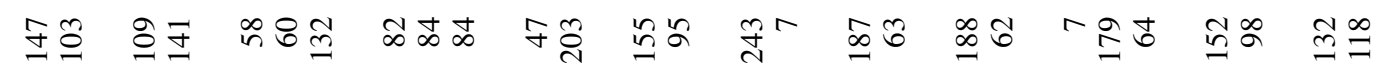
串在 吉吉 $\infty$

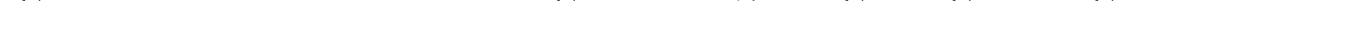


Table IV. Area under the receiver operating curve (AUC) and the corresponding 95\% CI of the combination of NSE, CEA, CA19-9, CA125 and CA242 (four different cancers versus healthy controls).

\begin{tabular}{|c|c|c|c|c|c|c|c|}
\hline Tumor markers & Sensitivity & Specificity & Youden index & AUC & SE & $95 \% \mathrm{CI}$ & P-value \\
\hline \multicolumn{8}{|c|}{ Colorectal cancer } \\
\hline NSE & 63.41 & 79.53 & 0.43 & 0.766 & 0.018 & $0.732,0.798$ & $<0.000$ \\
\hline CEA & 37.71 & 90.60 & 0.28 & 0.682 & 0.021 & $0.644,0.717$ & $<0.0001$ \\
\hline CA199 & 34.92 & 78.19 & 0.13 & 0.560 & 0.022 & $0.521,0.599$ & 0.007 \\
\hline CA125 & 25.14 & 88.93 & 0.14 & 0.590 & 0.022 & $0.552,0.688$ & $<0.0001$ \\
\hline CA242 & 66.20 & 59.73 & 0.30 & 0.651 & 0.021 & $0.613,0.688$ & $<0.0001$ \\
\hline Combination & 69.30 & 84.60 & 0.54 & 0.827 & 0.016 & $0.796,0.855$ & $<0.0001$ \\
\hline \multicolumn{8}{|l|}{ Rectum cancer } \\
\hline NSE & 69.95 & 77.18 & 0.47 & 0.794 & 0.021 & $0.755,0.829$ & $<0.0001$ \\
\hline CEA & 37.71 & 90.60 & 0.30 & 0.686 & 0.025 & $0.643,0.727$ & $<0.0001$ \\
\hline CA199 & 33.68 & 78.19 & 0.12 & 0.562 & 0.027 & $0.517,0.606$ & 0.0202 \\
\hline CA125 & 77.72 & 33.89 & 0.12 & 0.576 & 0.026 & $0.531,0.620$ & 0.0041 \\
\hline
\end{tabular}

NSE, neuron-specific enolase; CEA, carcinoembryonic antigen; CA199, cancer antigen 19-9; CA125, cancer antigen 125; CA242, cancer antigen 242; CRC, colorectal cancer; CI, confidence interval.

the 358 patients (CRC group) were compared with that of 298 healthy people (control group). It was revealed that the serum NSE levels in the CRC group were significantly higher compared with the control. Subsequently, the 358 patients comprising the CRC group were stratified according to the site of the tumor (specifically 193 rectum, 87 left colon and 78 right colon), and each was compared with the healthy control group. Notably, for each of these subgroups, the serum NSE level was significantly higher compared with the control group. This is in accordance with previous studies, which reported that serum NSE levels in patients with lung and non-colorectal tumors were markedly elevated compared with healthy participants (23). Further research is required to establish whether serum NSE levels may be used for CRC screening. In the present study, the serum levels of CEA, CA19-9, CA125 and CA242 were compared between the CRC and control groups, and it was determined that all serum marker levels were significantly higher in the CRC group, consistent with a previous study (35).

In addition, associations between each of the 5 markers in serum and certain clinicopathological parameters of CRC were investigated. It was demonstrated that the serum NSE level was significantly associated with pTNM staging, local lymphatic or distant metastasis. This indicates that serum NSE level may be as useful as CEA and other markers for the staging of patients with CRC, and NSE level may represent a precise indicator of local lymphatic or distant metastasis in CRC.

The cause of the difference in NSE levels between early and late clinical stages in CRC is yet to be elucidated. Perhaps, the NSE level is closely associated with (and may reflect the rate of) tumor growth. Enolase is a multifunctional protein, which is expressed abundantly in the cytosol. Upon stimulatory signals, enolase can locate to the cell surface and contribute to different pathologies, including injury, autoimmunity, infection, inflammation, and cancer (36). The present results warrant further experiments and follow-up investigation to confirm whether NSE is associated with tumor activity.
The sensitivity of CEA in the present study was $37.71 \%$, which is lower than has been previously reported (37). Previously, it has been revealed that patients who tested positive for serum CEA exhibited significantly different $\mathrm{T}, \mathrm{N}$ and $\mathrm{M}$ stages, tumor differentiation and pTNM staging (38). In the present study, preoperative positive CEA status was significantly associated with vascular invasion, nerve infiltration and tumor size. This indicated that CEA may be associated with CRC metastasis and tumor progression. Hence, preoperative serum CEA levels may predict CRC disease status (For example, tumor staging and lymph node metastasis) and provide a guide for a more informed clinical treatment strategy and prognosis. Furthermore, serum CEA level in patients with CRC has previously been identified as an independent predictor of both overall and disease-free survival time (39-41).

In the present study, serum CA242 status was significantly associated with the presence of $\mathrm{T}$ and $\mathrm{N}$ stages, nerve invasion and pTNM staging. This is consistent with previous studies that revealed that CA242 was a prognostic factor in patients with CRC (42). Similarly, in the present study serum CA19-9 and CA125 levels were significantly associated with lymph node metastasis and pTNM staging. The percentage of patients at stage III+IV who were positive for CA19-9 or CA125 was significantly higher compared with patients at stage I+II. These results are consistent with previous reports $(35,43)$. Taken together, these results suggest that CA19-9 and CA125 exhibit potential for the differential diagnosis, disease monitoring and therapeutic evaluation of numerous malignant tumors.

The majority of patients with CRC first present with blood in the stool as the primary symptom. Therefore, the association between tumor markers and hematochezia was analyzed in the present study. Of the 5 tumor markers tested, only NSE was associated with preoperative hematochezia. To the best of our knowledge, this finding has rarely been mentioned in other literature. It was hypothesized that the tumor growth and tissue characteristics of CRC result in symptoms of bloody stools accompanied by an elevated serum NSE level. Thus, 
examining the serum NSE of patients presenting with bloody stools may improve the diagnostic rate of CRC.

The accuracies of the AUC values $\geq 0.97,0.93-0.97$, 0.75-0.93 and $<0.75$ are considered excellent, very good, good and deficient or close to random, respectively (44). In the present study, in order to assess the diagnostic potential of NSE and other single-tumor biomarkers both individually and in combination, ROC curves were constructed and the corresponding AUCs were calculated. The AUC of NSE in patients with CRC was 0.766, and the AUCs stratified by tumor site were $0.794,0.777$ and 0.708 for rectal, left and right colon cancer, respectively. Thus, according to the AUC standard, serum NSE may represent an independent tumor biomarker $(A U C \geq 0.75)$. However, the accuracy of NSE alone for the diagnosis for CRC was not satisfactory. Therefore, other potential tumor markers (CEA, CA19-9, CA125 and CA242) were investigated in a similar manner.

The sensitivity and specificity of NSE in the diagnosis of CRC were 63.41 and $79.53 \%$, respectively, in the present study. Compared with the other four tumor markers commonly used, NSE is relatively reliable for the diagnosis of CRC. For the diagnosis of rectal colon cancer, the sensitivity and specificity of NSE were 69.95 and $77.18 \%$, respectively, and for left colon cancer they were 64.37 and $80.54 \%$, respectively. Compared with CEA, CA19-9 and CA125, NSE exhibits good sensitivity for the diagnosis of rectal cancer and CRC. However, for right colon cancer, although the sensitivity of NSE was only $44.87 \%$, the specificity was $91.28 \%$. Similarly, the sensitivity of CEA to CRC was only $37.71 \%$, and the sensitivity was $90.6 \%$. The aforementioned results are similar to those reported by McKeown et al (27), in which the reported specificity and sensitivity of CEA for CRC screening were 36.00 and $87.00 \%$, respectively. In the present study, it was calculated that CA242 indicated a good sensitivity for rectal, and left and right colon cancer (61.14-70.51\%), while the sensitivities of CA19-9 and CA125 fluctuated from 25.14 to $77.72 \%$, and the specificity was not precise.

In the present study, the AUCs of single tumor markers were significantly different between the patients overall and the subgroups according to tumor site. To improve diagnostic accuracy, 5 tumor biomarkers were combined using a logistic regression model. The combined markers in the logistic regression model exhibited a better diagnostic performance for CRC and the sensitivity of diagnosis was also better.

In conclusion, the present study revealed that serum NSE may represent an independent tumor biomarker for $\mathrm{CRC}$, and that it may be used for CRC auxiliary diagnosis. Furthermore, the combined detection of the tumor markers NSE, CEA, CA19-9, CA125 and CA242 was revealed as a significant combination of biomarkers that may be used in the diagnosis of CRC. The present study is limited in that the patients were all from a single center, the population size was small and follow-up information was lacking (since it is still ongoing). Therefore, whether NSE is a significant prognostic indicator for patients with CRC is yet to be elucidated. Nevertheless, the results of previous studies and the present study indicate that the association between NSE and CRC may be of great value in monitoring the recurrence of colorectal cancer and selecting adjuvant therapy in the foreseeable future.

\section{Acknowledgements}

The authors would like to thank Dr. Sun from Jilin University (Jilin, China) for providing statistical data analysis.

\section{Funding}

No funding was received.

\section{Availability of data and materials}

The datasets used and/or analyzed during the present study are available from the corresponding author upon reasonable request.

\section{Authors' contributions}

HL participated in the design of the experiment and the analysis of all clinical data as well as the revision of the paper. KS participated in the experimental design and paper modification. BL participated in the collection and analysis of pathological data. RL participated in the collection and analysis of serum markers. ZW participated in the collection and analysis of imaging data. ZX participated in data analysis and thesis revision. All authors read and approved the final manuscript.

\section{Ethics approval and consent to participate and}

The Ethics Standards Committee of China-Japan Union Hospital of Jilin University approved this study. All patients provided written informed consent form and the experiments were performed in accordance with the relevant guidelines and regulations of this hospital.

\section{Patient consent for publication}

Not applicable.

\section{Competing interests}

The authors have declared that they have no competing interests.

\section{References}

1. Stewart BW and Wild CP (eds): World Cancer Report 2014. IARC Press, Lyon, 2014.

2. Ferlay J, Soerjomataram I, Dikshit R, Eser S, Mathers C, Rebelo M, Parkin DM, Forman D and Bray F: Cancer incidence and mortality worldwide: Sources, methods and major patterns in GLOBOCAN 2012. Int J Cancer 136: E359-E386, 2015.

3. Siegel RL, Miller KD and Jemal A: Cancer statistics, 2016. CA Cancer J Clin 66: 7-30, 2016.

4. Marventano S, Forjaz M, Grosso G, Mistretta A, Giorgianni G, Platania A, Gangi S, Basile F and Biondi A: Health related quality of life in colorectal cancer patients: State of the art. BMC Surg 13 (Suppl 2): S15, 2013.

5. Helsingen LM, Vandvik PO, Jodal HC, Agoritsas T, Lytvyn L, Anderson JC, Auer R, Murphy SB, Almadi MA, Corley DA, et al: Colorectal cancer screening with faecal immunochemical testing, sigmoidoscopy or colonoscopy: A clinical practice guideline. BMJ 367: 15515, 2019

6. Weiss JB, Cetel NS and Weiss DE: To the Editor: Colorectal cancer screening: Colonoscopy has disadvantages. Cleve Clin J Med 86: 774-776, 2019. 
7. Wong CK, Fedorak RN, Prosser CI, Stewart ME, van Zanten SV and Sadowski DC: The sensitivity and specificity of guaiac and immunochemical fecal occult blood tests for the detection of advanced colonic adenomas and cancer. Int J Colorectal Dis 27: 1657-1664, 2012.

8. Van Rossum LG, van Rijn AF, Verbeek AL, van Oijen MG, Laheij RJ, Fockens P, Jansen JB, Adang EM and Dekker E: Colorectal cancer screening comparing no screening, immunochemical and guaiac fecal occult blood tests: A cost-effectiveness analysis. Int J Cancer 128: 1908-1917, 2011

9. Yan S, Han B, Gao S, Wang X, Wang Z, Wang F, Zhang J, Xu D and Sun B: Exosome-Encapsulated microRNAs as circulating biomarkers for colorectal cancer. Oncotarget 8: 60149-60158, 2017.

10. Cai J, Zuo X, Chen Z, Zhang Y, Wang J, Wang J, Ye X and Zhao W: Long noncoding RNAs serve as potential diagnostic biomarkers for colorectal cancer. J Cancer 10: 611-619, 2019.

11. Zhang H, Zhu M, Shan X, Zhou X, Wang T, Zhang J, Tao J, Cheng W, Chen G, Li J, et al: A panel of seven-miRNA signature in plasma as potential biomarker for colorectal cancer diagnosis. Gene 687: 246-254, 2019.

12. $\mathrm{Yu} \mathrm{H}$ : Reference intervals for gastrointestinal tumor markers (AFP, CEA, CA199 and CA724) in healthy adults of han nationality in chongqing by roche ECLIA system. Scand J Clin Lab Invest 79: 484-490, 2019.

13. Dong D, Zhang L, Jia L, Ji W, Wang Z, Ren L, Niu R and Zhou Y: Identification of serum periostin as a potential diagnostic and prognostic marker for colorectal cancer. Clin Lab 64: 973-981, 2018.

14. Song S, Hong JC, McDonnell SE, Koong AC, Minsky BD, Chang DT and Liauw SL: Combined modality therapy for rectal cancer: The relative value of posttreatment versus pretreatment CEA as a prognostic marker for disease recurrence. Ann Surg Oncol 19: 2471-2476, 2012

15. Tarantino I, Warschkow R, Schmied BM, Güller U, Mieth M, Cerny T, Büchler MW and Ulrich A: Predictive value of CEA for survival in stage I rectal cancer: A population-based propensity score-matched analysis. J Gastrointest Surg 20: 1213-1222, 2016.

16. Holdenrieder S, Dharuman Y, Standop J, Trimpop N, Herzog M, Hettwer K, Simon K, Uhlig S and Micallef J: Novel serum nucleosomics biomarkers for the detection of colorectal cancer. Anticancer Res 34: 2357-2362, 2014.

17. Webb A, Scott-Mackie P, Cunningham D, Norman A, Andreyev J, O'Brien $\mathrm{M}$ and Bensted $\mathrm{J}$ : The prognostic value of CEA, beta HCG, AFP, CA125, CA19-9 and C-erb B-2, beta HCG immunohistochemistry in advanced colorectal cancer. Ann Oncol 6: 581-587, 1995.

18. Wang J, Wang X, Yu F, Chen J, Zhao S, Zhang D, Yu Y, Liu X Tang $\mathrm{H}$ and Peng $\mathrm{Z}$ : Combined detection of preoperative serum CEA, CA19-9 and CA242 improve prognostic prediction of surgically treated colorectal cancer patients. Int J Clin Exp Pathol 8: 14853-14863, 2015.

19. Isgrò MA, Bottoni $P$ and Scatena R: Neuron-Specific enolase as a biomarker: Biochemical and clinical aspects. Adv Exp Med Biol 867: 125-143, 2015

20. Barone FC, Clark RK, Price WJ, White RF, Feuerstein GZ, Storer BL and Ohlstein EH: Neuron-specific enolase increases in cerebral and systemic circulation following focal ischemia Brain Res 623: 77-82, 1993.

21. Muller E, Shock JP, Bender A, Kleeberger J, Högen T, Rosenfelder M, Bah B and Lopez-Rolon A: Outcome prediction with serial neuron-specific enolase and machine learning in anoxic-ischaemic disorders of consciousness. Comput Biol Med 107: 145-152, 2019.

22. Schmechel D, Marangos PJ and Brightman M: Neurone-Specific enolase is a molecular marker for peripheral and central neuroendocrine cells. Nature 276: 834-836, 1978.

23. Xu L, Lina $\mathrm{W}$ and Xuejun Y: The diagnostic value of serum CEA, NSE and MMP-9 for on-small cell lung cancer. Open Med (Wars) 11: 59-62, 2016.

24. Amin MB, Greene FL, Edge SB, Compton CC, Gershenwald JE Brookland RK, Meyer L, Gress DM, Byrd DR and Winchester DP: The Eighth Edition AJCC Cancer Staging Manual: Continuing to build a bridge from a population-based to a more 'personalized' approach to cancer staging. CA Cancer J Clin 67: 93-99, 2017

25. Qi W, Li X and Kang J: Advances in the study of serum tumor markers of lung cancer. J Cancer Res Ther 10: C95-C101, 2014.
26. Liu L, Xu HX, Wang WQ, Wu CT, Xiang JF, Liu C, Long J, $\mathrm{Xu}$ J, Fu de L, Ni QX, et al: Serum CA125 is a novel predictive marker for pancreatic cancer metastasis and correlates with the metastasis-associated burden. Oncotarget 7: 5943-5956, 2016.

27. McKeown E, Nelson DW, Johnson EK, Maykel JA, Stojadinovic A, Nissan A, Avital I, Brücher BL and Steele SR: Current approaches and challenges for monitoring treatment response in colon and rectal cancer. J Cancer 5: 31-43, 2014.

28. Campos-da-Paz M, Dórea JG, Galdino AS, Lacava ZGM and de F atima Menezes Almeida Santos M: Carcinoembryonic Antigen (CEA) and hepatic metastasis in colorectal cancer: Update on biomarker for clinical and biotechnological approache. Recent Pat Biotechnol 12: 269-279, 2018.

29. Sung HJ and Cho JY: Biomarkers for the lung cancer diagnosis and their advances in proteomics. BMB Rep 30: 615-625, 2008.

30. Gao Y, Wang J, Zhou Y, Sheng S, Qian SY and Huo X: Evaluation of serum CEA, CA19-9, CA72-4, CA125 and ferritin as diagnostic markers and factors of clinical parameters for colorectal cancer. Sci Rep 8: 2732, 2018.

31. Wang P, Piao Y, Zhang X, Li W and Hao X: The concentration of CYFRA 21-1, NSE and CEA in cerebro-spinal fluid can be useful indicators for diagnosis of meningeal carcinomatosis of lung cancer. Cancer Biomark 13: 123-130, 2013.

32. Wang JL, Wu DW, Cheng ZZ, Han WZ, Xu SW and Sun NN: Expression of high mobility group box-B1 (HMGB-1) and matrix metalloproteinase-9 (MMP-9) in non-small cell lung cancer (NSCLC). Asian Pac J Cancer Prev 15: 4865-4869, 2014.

33. Kaiser E, Kuzmits R, Pregant P, Burghuber O and Worofka W: Clinical biochemistry of neuron specific enolase. Clin Chim Acta 183: 13-31, 1989.

34. Yang WY, Shi G, Wu LP, Wei ST, Huang YN, Tan LX, Yang RZ, Yan CX, Guo ET, Wang HY, et al: Analysis of specific serum markers of colon carcinoma using a bhattacharyya-based support vector machine. Genet Mol Res 23: 16, 2017.

35. Ning S, Wei W, Li J, Hou B, Zhong J, Xie Y, Liu H, Mo X, Chen J and Zhang L: Clinical significance and diagnostic capacity of serum TK1, CEA, CA 19-9 and CA 72-4 levels in gastric and colorectal cancer patients. J Cancer 9: 494-501, 2018.

36. Haque A, Ray SK, Cox A and Banik NL: Neuron specific enolase: A promising therapeutic target in acute spinal cord injury. Metab Brain Dis 31: 487-495, 2016.

37. Fakih MG and Padmanabhan A: CEA monitoring in colorectal cancer. What you should know. Oncology (Williston Park) 20: 579-587, 2006

38. Baqar AR, Wilkins S, Staples M, Angus Lee CH, Oliva K and McMurrick P: The role of preoperative CEA in the management of colorectal cancer: A cohort study from two cancer centres. Int J Surg 64: 10-15, 2019.

39. Takagawa R, Fujii S, Ohta M, Nagano Y, Kunisaki C, Yamagishi S, Osada S, Ichikawa Y and Shimada H: Preoperative serum carcinoembryonic antigen level as a predictive factor of recurrence after curative resection of colorectal cancer. Ann Surg Oncol 15: 3433-3439, 2008

40. Wang WS, Lin JK, Chiou TJ, Liu JH, Fan FS, Yen CC, Lin TC, Jiang JK, Yang SH, Wang HS and Chen PM: Preoperative carcinoembryonic antigen level as an independent prognostic factor in colorectal cancer: Taiwan experience. Jpn J Clin Oncol 30: 12-16, 2000.

41. Wiratkapun S, Kraemer M, Seow-Choen F, Ho YH and Eu KW: High preoperative serum carcinoembryonic antigen predicts metastatic recurrence in potentially curative colonic cancer: Results of a five-year study. Dis Colon Rectum 44: 231-235, 2001.

42. Yang XQ, Chen C, Hou JX, Peng CW, Huang CQ and Li Y: Preoperative serum carbohydrate antigen 242 is a useful predictive and prognostic marker in colorectal cancer. Hepatogastroenterology 58: 377-382, 2011.

43. Okamura R, Hasegawa S, Hida K, Hoshino N, Kawada K, Sugihara K and Sakai Y; Japanese Study Group for Postoperative Follow-up of Colorectal Cancer: The role of periodic serum CA19-9 test in surveillance after colorectal cancer surgery. Int J Clin Oncol 22: 96-101, 2017.

44. Li H, Zhang B, Hu X, Dong Y, Fan Q, Guo F, Ren X, Zhou H, Tian W and Zhao Y: Serum helicobacter pylori FliD antibody and the risk of gastric cancer. Oncotarget 7: 22397-22408, 2016.

This work is licensed under a Creative Commons Attribution-NonCommercial-NoDerivatives 4.0 International (CC BY-NC-ND 4.0) License. 